\title{
Phase-Contrast-Based Structure Retrieval Methods in Atomic Resolution Scanning Transmission Electron Microscopy - When They Hold and When They Don't
}

Scott Findlay ${ }^{1}$, Leslie Allen ${ }^{2}$, Hamish Brown $^{3}$, Zhen Chen ${ }^{4}$, Jim Ciston $^{3}$, Yuichi Ikuhara ${ }^{5}$, Ryo Ishikawa ${ }^{5}$, Colin Ophus ${ }^{3}$, Gabriel Sánchez-Santolino ${ }^{6}$, Takehito Seki ${ }^{5}$, Naoya Shibata ${ }^{5}$ and Matthew Weyland ${ }^{1}$

${ }^{1}$ Monash University, Clayton, Victoria, Australia, ${ }^{2}$ The University of Melbourne, Parkville, Victoria, Australia, ${ }^{3}$ Lawrence Berkeley National Laboratory, Berkeley, California, United States, ${ }^{4}$ Cornell University, Ithaca, New York, United States, ${ }^{5}$ University of Tokyo, Bunkyo-ku, Tokyo, Japan, ${ }^{6}$ Universidad Complutense de Madrid, Madrid, Madrid, Spain

The development and proliferation of segmented and pixelated detectors suitable for high-resolution scanning transmission electron microscopy (STEM) has seen a resurgence of phase-contrast-based structure retrieval methods, including differential phase contrast [1-4] and various forms of ptychography [5-9]. Using most of the scattering distribution makes these methods dose efficient [3,7], enabling application to ultrathin samples [8-11]. Such thin samples offer another advantage - they best justify the (strong) phase object approximation underpinning these methods. In this approximation, scattering through the sample is mathematically described as a product of the incident wavefield with a transmission function. The phase of this transmission function - proportional to the projected electrostatic potential of the sample - is what phase-contrast-based structure retrieval methods seek to determine. While qualitatively reasonable retrievals (e.g. giving good indication of column locations) can be achieved for samples tens of nanometers thick, for more quantitative work, such as measuring electron redistribution due to bonding, the phase object approximation can start to break down beyond a few nanometers in sample thickness $[12,13]$.

One reason the phase object approximation is restrictive in STEM is that it assumes all scattering occurs in a single plane, thus neglecting all spatial propagation of the wavefield within the sample including geometric probe spreading. In cases where geometric probe spreading dominates, structure retrieval might be attempted using the thick weak phase object approximation which includes full spatial propagation at the expense of including only single scattering from the electrostatic potential. For sufficiently large probe forming apertures, simulations predict this can be a good approximation even in samples containing heavy elements [14]. But if both multiple scattering and spatial propagation are significant, quantitatively reliable structure retrieval requires handling dynamical diffraction. It was recently shown that fast-readout pixel detectors offer a route to recording sufficient scattering data to effect such a reconstruction via inversion of the so-called scattering matrix [15].

This talk will explore how developments in segmented and pixelated detectors facilitate phase-contrastbased structure retrieval methods, and explore their domains of validity. Figure 1 compares the scattering matrix ("S-matrix") method, differential phase contrast (DPC) and the thick weak phase object approximation (thick WPOA) for three different thicknesses. Plots in the upper half represent Fourier coefficients of the reconstruction, emphasizing that low order Fourier coefficients (potentially containing bonding information) are more reliably reconstructed than those of high order. Images in the lower half show real space potentials, emphasizing the qualitative appearance of the reconstructions (though the range is sensitive to small errors in Fourier coefficients). These are reconstructions from ideal, simulated scattering data, but we shall further discuss how noise and other practical constraints limit the reliability of these methods [16]. 

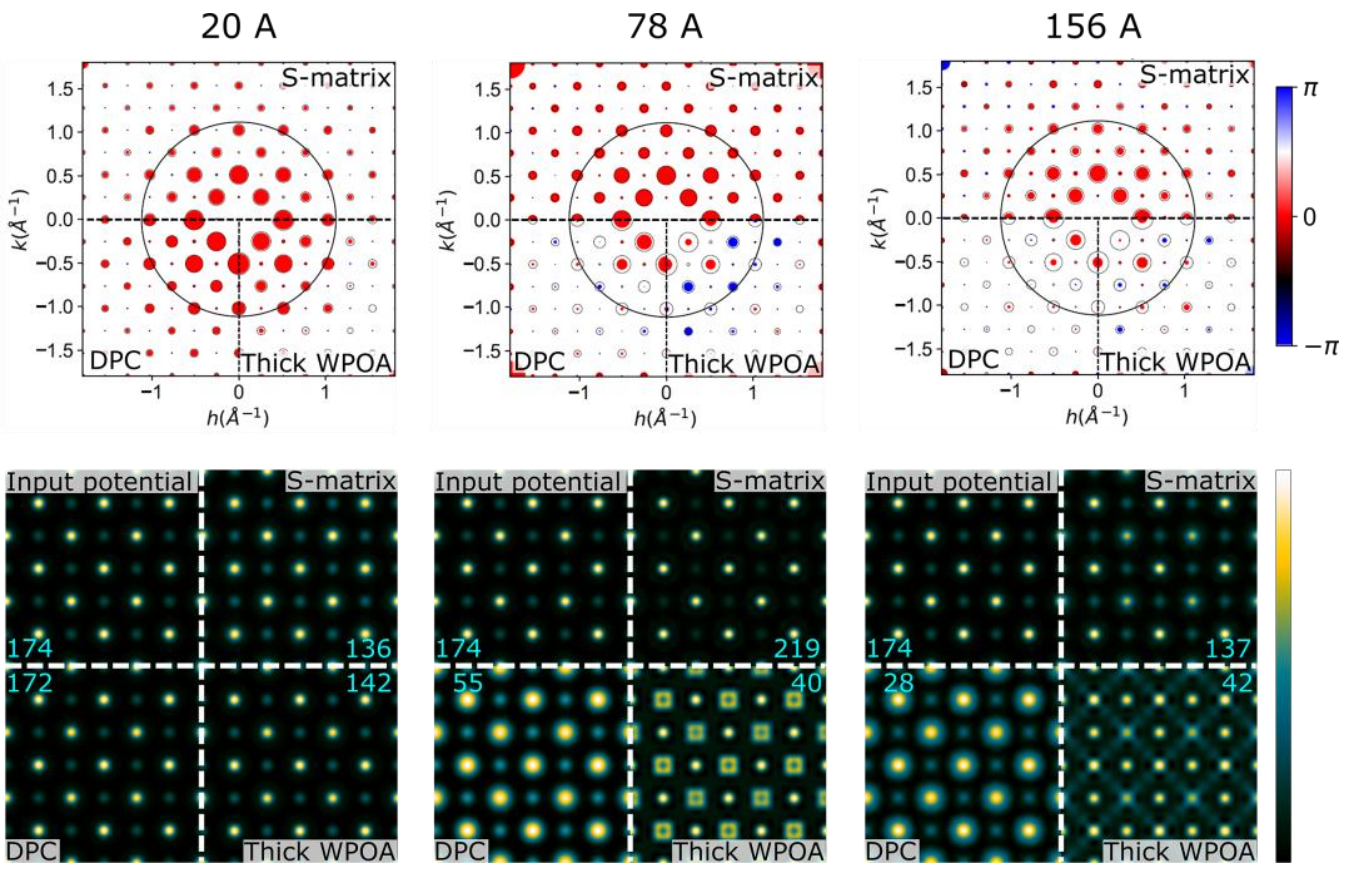

Figure 1. Comparing structure retrieval methods (on simulated scattering data) for $\mathrm{SrTiO}_{3}$ [001] sample thicknesses of $20 \AA, 78 \AA$ and $156 \AA$. Upper row: Fourier coefficients of the potential. Small black circles have area proportional to the magnitude of the Fourier coefficient of the input potential for the spatial frequency on which the circle is centred. The colored disks have area proportional to the magnitude of the reconstructed Fourier coefficients. Color reflects the phase difference between reconstructed and reference Fourier coefficients. Lower row: projected potentials in real space. Each is shown normalized on its own color scale, with the range in volts being inset in cyan text on each image.

\section{References}

[1] N Shibata et al., Nature Physics 8 (2012) 611.

[2] K Müller et al., Nature Communications 5 (2014) 5653.

[3] I Lazić, EGT Bosch, S Lazar, Ultramicroscopy 160 (2016) 265.

[4] W Gao et al., Nature 575 (2019) 480.

[5] CT Putkunz et al., Phys. Rev. Lett. 108 (2012) 073901.

[6] A Morgan et al. Phys. Rev. B 87 (2013) 094115.

[7] TJ Pennycook et al., Ultramicroscopy 151 (2015) 160; H Yang, TJ Pennycook, PD Nellist, Ultramicroscopy 151 (2015) 232.

[8] H Yang et al., Nature Communications 7 (2016) p. 12532.

[9] Y Jiang et al., Nature 559 (2018) p. 343.

[10] G Sánchez-Santolino et al., ACS Nano 12 (2018) 8875.

[11] R Ishikawa et al., Nature Communications 9 (2018) 3878.

[12] R Close et al., Ultramicroscopy 159 (2015) 124.

[13] K Müller-Caspary et al., Ultramicroscopy 178 (2017) 62.

[14] HG Brown et al., Ultramicroscopy 197 (2019) 122.

[15] HG Brown et al., Phys. Rev. Lett. 121 (2018) 266102.

[16] This research was supported under the Australian Research Council Discovery Projects funding scheme (Projects No. DP160102338 and FT190100619). Work at the Molecular Foundry was supported by the Office of Science, Office of Basic Energy Sciences, of the U.S. Department of Energy under Contract No. DE-AC02$05 \mathrm{CH} 11231$. 